\title{
Briefing: High-performance computing for city-scale modelling and simulations
}

Kenichi Soga FREng, FICE

Chancellor's Professor, University of California, Berkeley, CA, USA

(corresponding author: soga@berkeley.edu)

Gerard Casey MSc

PhD Student, University of Cambridge, Cambridge, UK; Arup, London, UK
Krishna Kumar PhD

Research Associate, University of Cambridge, Cambridge, UK

Bingyu Zhao MSc

PhD Student, University of Cambridge, Cambridge, UK

Smarter cities and infrastructure are essential if the world is to respond effectively to the critical challenges it faces. As of 2008, and for the first time in human history, more than half of the world's population now live in cities. Yet cities increasingly need to be able to do more with less, to provide for the well-being of their citizens in a truly sustainable way. City-scale simulations are becoming a possibility with advancements in the computing hardware and software technologies. Recent advancements in distributed scaling using Apache Spark is exemplified through a case-study on simulating the traffic flow behaviour of London at per-hour time resolution. The case-study on the response of ground and structures in central Tokyo shows the advancements in multigrid techniques and Finite Element simulations. This paper discusses the advancements and challenges in high-performance computing (hardware, algorithms, software, and visualisation techniques) in achieving the possibility of real-time city-scale simulations.

\section{City-scale models and simulations}

The twenty-first century is witnessing a rapid rise of urbanisation in both the developed and the developing worlds. Cities increasingly need to be able to do more with less in order to provide for the wellbeing of their citizens in a sustainable way. The promise of a smart city is an emerging ability to understand, to respond to and to shape human activity at urban populations and geographic scales so that a more agile, adaptive and sustainable urban environment can be created (see Batty et al., 2012; Caragliu et al., 2011; Chourabi et al., 2012; Kitchin, 2014; Su et al., 2011 for the early adoption of a smart city). To be effective, this requires the predictive power of datadriven modelling and city-scale computational simulations. Recently, city-scale simulations are becoming possible thanks to a surge of development in the high-performance computing (HPC) domain including advanced hardware and computational and algorithmic techniques such as domain decomposition across multigraphic processing units (multi-GPUs) and multigrid techniques. Advanced HPC systems (quadrillion floating point operations per second) are now becoming available for performing city-scale simulations with microscale models of an individual objective (structure, people, vehicle etc.) (e.g. Hori, 2011; Johansen et al., 2017; Lu and Guan, 2017; Pijanowski et al., 2014; Sánchez-Medina et al., 2010; Wijerathne et al., 2013; Yoshimura et al., 2016; Zia et al., 2012).

Agent-based modelling ( $\mathrm{ABM}$ ) is perhaps the most promising modelling paradigm for attempting to understand complex city systems and facilitating a range of different scenario-testing exercises (Casey et al., 2017). Figure 1 shows the visualisation of the graph-parallel distributed computing tool that simulates city-scale infrastructure networks with a hundred thousand links and millions of agents traversing close to real time. The tool performs data analytics on the results from ABM simulations and monitoring data. Transport services are modelled as edges, and the interaction points (e.g. bus stops) are modelled as vertices in a graph network. Real- time and historic large data sets are used to initialise and update the behaviour of the infrastructure and the agents. The purpose of the $\mathrm{ABM}$ tool is to capture the complex city-scale response from individual agent behaviours. Macroscale events such as earthquakes influence the weights of the edges on a graph network (e.g. reduced road capacity/road closures update the weights of the edges/removal of an edge), which in turn affect the behaviour of individual agents, changing the response of a city. The real-time sampling feature allows for querying and updating individual agent behaviours in a scenario testing of global impacts, such as in understanding the resilience of a city to an earthquake scenario.

Figure 2(a) shows a city-scale model of London with 300000 nodes and traffic levels at 8 a.m. on a typical weekday. Five million agents are simulated at a time resolution of $1 \mathrm{~h}$ to traverse the city using various origin-destination pairs using HPC. Road capacity can be decreased, or a road can be removed to model infrastructure failure, as shown in Figure 2(b).

Another example of a city-scale computing tool is the highresolution finite-element modelling of ground motion to analyse the response of structures during an earthquake. Yoshimura et al. (2016) performed seismic wave amplification simulation for an urban area of $2.0 \times 2.0 \mathrm{~km}^{2}$ in central Tokyo; this region consists of businesses, shops and densely built residential districts, with a total of 13275 buildings. The ground structure has a rectangular shape of $2 \cdot 0 \times 2 \cdot 0 \mathrm{~km}^{2}$ and a depth of $100 \mathrm{~m}$. The ground structure is modelled with soil layers using the $5 \mathrm{~m}$ grid elevation map and digital geotechnical database. Figures 3(a), 3(c) and 3(d) show the response of the ground at the surface as well as the response of the modelled structures. Figure 3(b) shows that ground motion responses are far from being uniform and the resulting structure shaking changes. Such a complexity in the coupled seismic-structure response distribution in an urban area can be 
Smart Infrastructure and Construction Volume 170 Issue SC4
Briefing: High-performance computing

for city-scale modelling and simulations

Soga, Casey, Kumar and Zhao

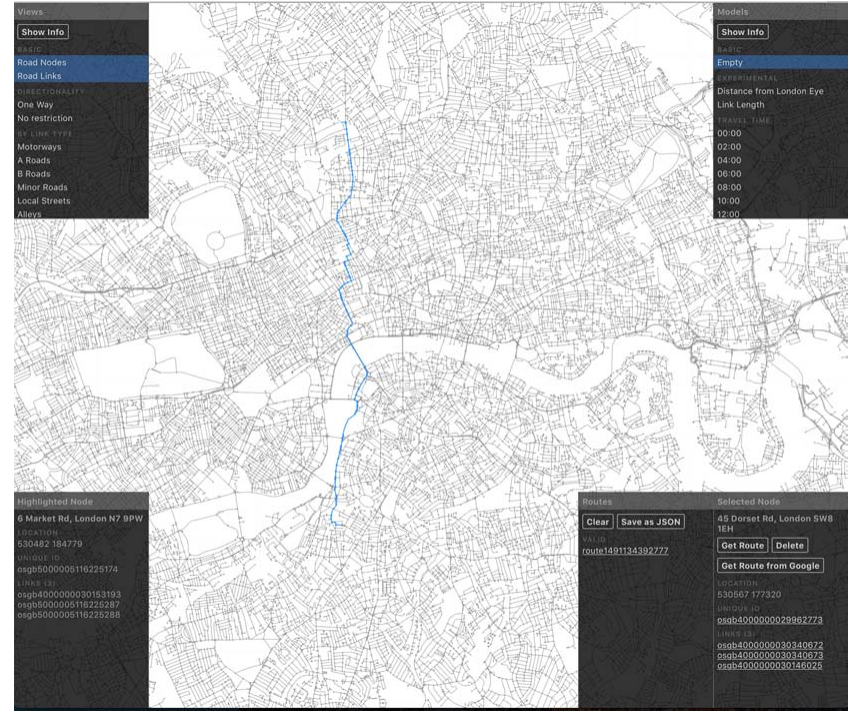

Figure 1. An example shortest path query for travel by car

realised only by combining high-resolution three-dimensional (3D) ground motion analysis and structure response analysis using HPC.

\section{2. $\mathrm{HPC}$}

For the city-scale modelling of interactions among people, infrastructure and environment in natural or man-made disasters using HPC, each component of a city has different characteristics and computational requirements (in terms of spatial and temporal

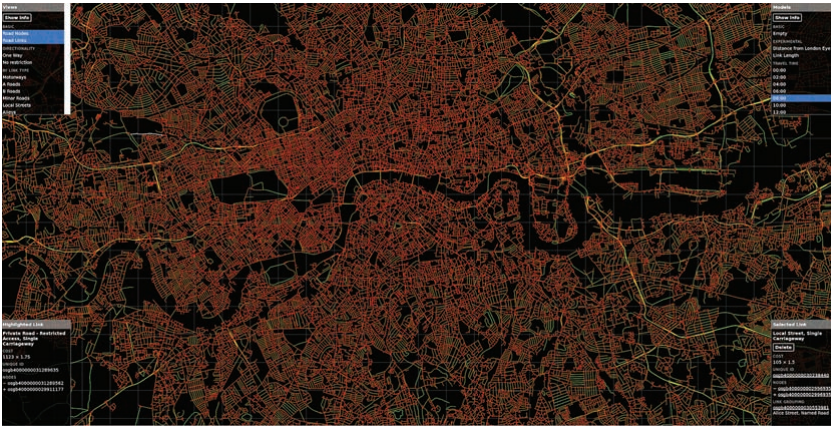

(a)

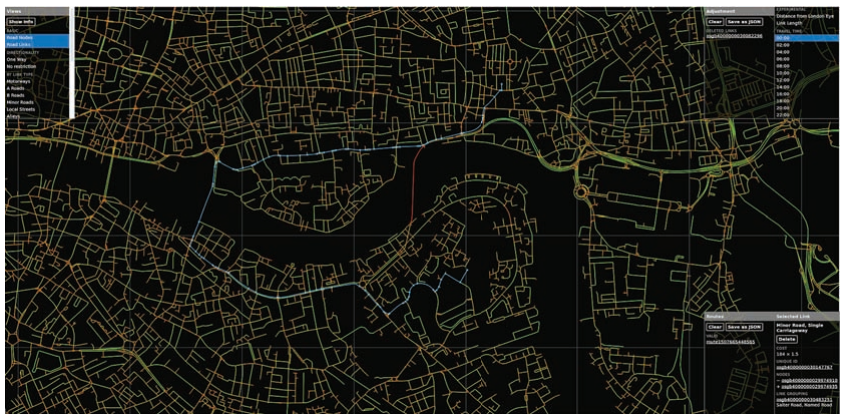

(b)

Figure 2. City-scale ABM tool. (a) Browser-based visualisation of journey times on a typical working day at 8 a.m. in London. (b) Microimpact assessment of a bridge closure at midnight in London

resolutions, spatial dimension, computational tool etc.) (Tadokoro et al., 2000).

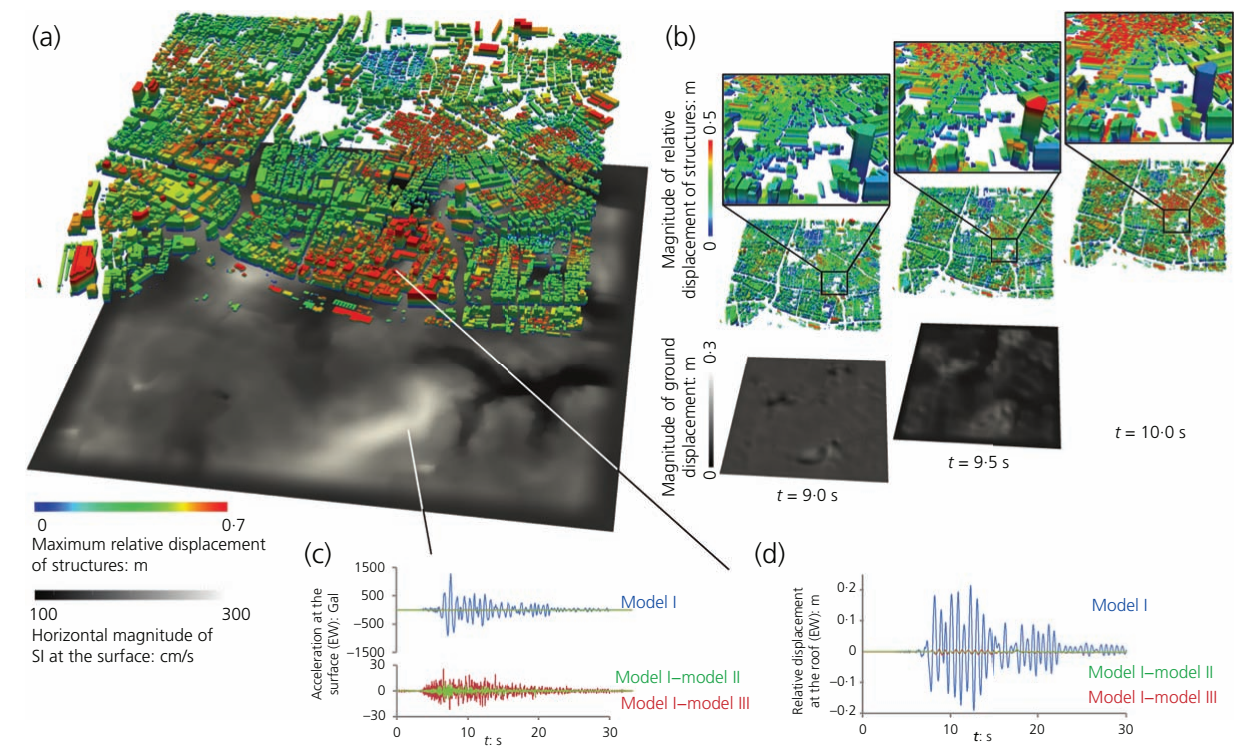

Figure 3. Response of ground and structures in central Tokyo. (a) Horizontal magnitude of spectral intensity (SI) at the surface and maximum relative displacement of structures. (b) Snapshots of time history response. (c) Acceleration waveforms at the surface (point A) obtained from model I (10.7 billion degrees of freedom model), model II (with element sizes 1.5 times larger than model I) and model III (with element sizes three times larger than model I). (d) Relative displacement waveforms of structure A (a two-storey reinforced concrete building located above point A) obtained using acceleration waveforms computed using models I, II and III). EW, east-west 
The computation performance of an HPC program depends on the hardware, the modelling paradigm and the software implementation. Until a decade ago, the speed of processors, following Moore's observation, has doubled approximately every 18 months, when the technology hit the energy consumption and the heat dissipation wall (Waldrop, 2016). The HPC community moved towards multicore architecture and was revolutionised by the appearance of general-purpose GPUs (GPGPUs). Several large supercomputers now use GPUs as accelerators. For example, the Cray Titan supercomputer at the Oak Ridge National Laboratory, the USA's fastest supercomputer in Top500 - November 2016, has been accelerated by Nvidia Kepler GPUs. The latest Nvidia Pascal GPUs have bidirectional interconnect accelerating the bandwidth. In 2012, Intel announced the Many Integrated Core (MIC) architecture product - the Xeon Phi coprocessor, which is highly parallel and can be used to accelerate general-purpose computing. Xeon Phi uses a symmetric multiprocessor on a chip that has cache coherence and uniform memory access. The National Energy Research Scientific Computing Center Cori (Cray XC40), ranked fifth in the Top500, is accelerated using Intel Xeon Phi coprocessors (Top500, 2017).

Over the last 25 years, message passing interface (MPI), an HPC standard for efficiently communicating the layout of data in memory over the network, has supported a substantial majority of supercomputing work. With the advent of MIC and GPGPUs, there is a need to improve the performance on a single node and to utilise efficiently all available compute cores. Shared memory multiprocessing paradigms such as Open Multiprocessing, Open Accelerators and Intel Threading Building Blocks have gained traction in the world of traditional HPC. As compilers become efficient in identifying vectorisable regions of codes, there is an increased focus in compiler autoparallelisation. Parallel programming frameworks such as Kokkos and Charm++ focus on improving performance portability across multiple shared and distributed memory architectures (Edwards et al., 2014; Kale and Krishnan, 1993).

As the complexity of research tools increases, the development environment and dependencies are critical for reproducibility. In the past few years, there has been an increased adoption of container and orchestration technologies such as Docker, Singularity and Kubernetes. Containers wrap up a piece of software in a complete file system that contains everything it needs to run: code, runtime, system tools, system libraries - anything one can install on a server. This guarantees that a container will always run the same, regardless of the environment it is deployed to (Bernstein, 2014). Singularity (2017) is an implementation of a container and is an engine for running containers without requiring any privileged access (root access). Containers allow researchers to isolate the software environment needed to produce a result away from the configuration and operating system of the computer that the analysis will be running on. As the computing jobs become more complex, resource management and scheduling tools such as Yarn and Mesos become increasingly relevant.

\section{Data management}

Internet-scale data result in high computational demands. Solving a big-data problem in HPC is challenging. In HPC, large and homogenous arrays are read from a parallel disc and processed with symmetric resources. On the other hand, big data are often heterogeneous and unstructured data, often available in real time, located in a distributed setting and processed with asymmetric resources. In the mid-2000s, analysing and interpreting real-time streaming data became mainstream along with scalability, largescale data storage, distributed computing and fault tolerance (Chen et al., 2014; Hashem et al., 2015). Traditional MPI-based applications had the wrong level of abstraction for input/output (I/O)-intensive/fault-tolerant applications. Message-passing-based frameworks soon became very important platforms for building complex parallel and distributed software in different communities. For example, Erlang (2017), released to the public just 5 years later, is a functional language with built-in message passing that has played a very large role in many communications and control environments. Akka (2017) is a Scala-based message passing framework which supports distributed data model and cluster sharding for processing transactions of big data. Apache Spark (2017) is a general-purpose cluster engine for big data processing with a resilient distributed data set (RDD) capable of supporting graph frameworks. Spark optimises the tasks running on each file according to its size to utilise the cluster fully. Fundamentally, the RDDs are stored in partitions and are operated in parallel using cached data in-memory, which allows for multiple operations across the same data. Spark is often used alongside Hadoop's data storage module (Hadoop Distributed File System). Hadoop as a big-dataprocessing technology has been around since 2011 and has proven to be the solution of choice for processing large data sets. Hadoop offers distributed fault-tolerant and persistent distributed file system and data recovery capabilities (Hashem et al., 2015).

The emergence of big data has led to a shift in how data are stored, processed and analysed. Modern graph-processing systems apply a vertex-centric logic to transform data on a graph and exploit the graph structure to achieve more efficient distributed execution (Malewicz et al., 2010). Graphs are ubiquitous; the volume and diversity of huge graphs with billions of entities and relationships are a driving force for the development of powerful and highly parallel big-data systems such as GraphX (2017). Graph database systems consider graphs as first-class citizens in their data model, query languages and application programming interfaces (APIs). They also provide the ability to insert, delete and modify data in a graph database using transactional semantics in addition to running global algorithms on the network, such as shortest-path algorithms, as well as implement appropriate integrity constraints over the graph (Angles, 2012; Angles and Gutierrez, 2008; Vicknair et al., 2010). However, graph algorithms such as shortest path queries remain mathematically hard problems. GPU and algorithmic implementations show the most promise to solve these problems.

Traditional structured query language (SQL) database poses scalability issues when querying massive geospatial data sets. In 
Briefing: High-performance computing

for city-scale modelling and simulations

Soga, Casey, Kumar and Zhao order to parallelise data processing, a lightweight data interchange format, JavaScript Object Notation can be used to distribute data across the computing nodes (Cattell, 2010). The data processing, when done in-memory, is not restricted by the constant reading and writing of results to a database. This enables a simulation of large-scale complexity to be possible in a reasonable time frame. Elasticsearch (2017) is a distributed search engine capable of performing near-real-time searches, by using shared indices with replicas distributed across several computing nodes. Data serialisation frameworks such as Apache Avro (2017) improves the efficiency of data processing (Palmer et al., 2011). while frameworks such as Apache Cassandra (2017), a highly available, linearly scalable data store, is optimised for writing-intensive workloads (Chebotko, 2015).

City-scale simulations require storing large quantities of a diverse data set. Hierarchical Data Format 5 (HDF5, 2017) is an I/O middleware library and file format for storing and managing data. HDF5 supports an unlimited variety of data types and is designed for flexible and efficient $\mathrm{I} / \mathrm{O}$ and for high-volume and complex data (Folk et al., 2011). MPI implementations are good for optimising various access patterns in applications, and the MPI standard supports complex data types for scientific data. However, MPI does not store any 'metadata' for applications, such as the data types themselves, the dimensionality of an array and the names for specific objects. HDF5, therefore, offers the ability to handle data at a higher level, thus allowing parallel I/O methods. With remoteprocedure call, which allows systems to request services located on other machines through the network, HDF5 calls on one machine can be executed on another. Tiered-storage architectures with 'burst buffers' are gaining more traction, which will be the norm for systems. HDF5 will need to undergo major changes to the data model and file format to leverage such systems fully. Research incorporating concepts such as data movements, transactional I/O, indexing and asynchrony to HDF5 for HPC is ongoing.

\section{Visualisation}

Real-time visualisation of scientific city-scale simulations is a necessity. I/O in HPC application has long been a bottleneck. Accessing, extracting and assessing the scientific content of largescale simulation data sets, at the terabyte scale, require specialised tools and techniques. It is not always meaningful to post-process a simplified version of the data. In situ visualisation libraries such as Icarus and ParaView Catalyst (2017) examine and query regions of interest and also render simulations in real time. A loosely coupled and push-driven approach is adopted to link simulation and visualisation using a shared memory-mapped file as an interconnect. This technology allows for transparent data communication across the network using a client (simulation)/ server (post-processing) model using sockets or MPI layers, thus enabling the ability to pause, examine, slice and visualise realtime simulations (Fabian et al., 2011).

Visualisation of complex networks with spatio-temporal information always poses a complex challenge. A web-based interface using Web
Graphics Library (WebGL) and JavaScript API provides crossplatform functionality. WebGL (Mozilla, 2017) is a cross-platform, low-level JavaScript API based on Open Graphics Library for rendering two-dimensional and 3D graphics in any compatible browsers without the need for plug-ins. CityGML is an extensiblemarkup-language-based open standardised data model and exchange format to store digital 3D models of cities and landscapes. CityDB (2017) is an open-source 3D city database built on CityGML for 3D city visualisation on the Web using a JavaScript API. Frameworks such as Open Street Map (2017) and LeafletJS (2017) provide the ability to display data sources and simulation results on city-scale models using an open standard. Advanced ray-tracing algorithms, such as those used in Disney's Hyperion rendering engine, are able to simulate and visualise cities without the need to downsample or remesh the city (Eisenacher et al., 2013).

\section{Challenges}

There are two challenges for large-scale simulations using HPC: (a) scalable algorithms and (b) optimisation of $\mathrm{I} / \mathrm{O}$ through data parallelism.

Algorithms are no longer merely required to communicate across compute nodes, but to couple efficiently with shared memory resources within each node. Sustainable future-proof computing algorithms have to be performance-portable so that they can efficiently run on various current and future memory-caching layouts and computing architectures.

Data parallelism is almost always I/O intensive (more than 50\% and, in some cases, 98\%). The amount of data to visualise is typically of the order of total memory. There are two main factors in visualising large data: $(a)$ how much data that need to be read and $(b)$ how fast they can be read. Relative $\mathrm{I} / \mathrm{O}$, which is the ratio of the total memory to the $\mathrm{I} / \mathrm{O}$, is quickly becoming a dominant cost in the supercomputing application. In situ data processing, coupling visualisation and analysis routines with the simulation code (no I/O) will enable large-data visualisation. A tightly coupled system of synchronous coprocessing is highly constrained by the memory, impacts performance and is prone to crashes. However, a loosely coupled system requires knowing the regions of interest beforehand. A hybrid approach means that the data are reduced to a tightly coupled setting (visualisation and analysis have direct access to the memory of simulation code) and are sent to asynchronous concurrent resources. The Scalable Data Management, Analysis and Visualization Institute at the Lawrence Berkeley National Laboratory is developing in situ processing and parallel I/O frameworks to enable software-based visualisation on large-scale computing facilities.

A multilayered graph could be used to model different levels of the system, such as the infrastructure networks and the relationship between these different layers, using real-time and historic large data sets. For example, the London transport graph consists of 423541 links at the atomic level. There are various sources of data for city-scale simulations. Dynamic streaming data have to be 
Smart Infrastructure and Construction

Volume 170 Issue SC4
Briefing: High-performance computing

for city-scale modelling and simulations

Soga, Casey, Kumar and Zhao processed in real time; examples include a dynamically computed general transit feed specification considering real-time feeds at a rate of $24 \mathrm{~GB} / \mathrm{d}$, public transit data sourced at $30 \mathrm{~s}$ intervals from Transport for London and vehicular traffic data sourced at $2 \mathrm{~h}$ intervals with 200000 API requests at a rate of $1 \mathrm{~GB} / \mathrm{d}$. Other data inputs such as survey, census information and routing schedules are passive sources and are typically batch-processed. Crowdsourced data, which include social network and transport data, are available at such scale, and the efficient use of these data sources to analyse and predict the behaviour of smart cities requires asynchronous, concurrent processing on large-scale HPC systems.

\section{Closure}

The idea that more information in more dynamic and iterative ways gives better prediction of infrastructure status, transportation behaviour and people movement is central to the promise of the progression of city-scale models using HPC. It is hypothesised that through new data sources such as remote sensors and mobile phones, the reliance on heavily simplified generalised functions for model inputs will be erased. This trade-off between idealised and actual empirical data will be matched with dynamic models that consider complexity at a fundamental level, inherently mirroring the systems they are attempting to replicate. HPC brings the possibility of doing all of these in less time than the simplified crude models of the past. The hope is to have better answers at the time of important decisionmaking junctures. Whether this hypothesis is true or not in realising the concept of smart infrastructure and city remains to be seen, but this initiative is worth pursuing.

\section{REFERENCES}

Akka (2017) https://akka.io (accessed 29/11/2017).

Angles R (2012) A comparison of current graph database models. In Proceedings of the 2012 IEEE 28th International Conference on Data Engineering Workshops (Kementsietsidis A and Vaz Salles M (eds)). Institute of Electrical and Electronics Engineers, Washington, DC, USA, pp. 171-177.

Angles R and Gutierrez C (2008) Survey of graph database models. ACM Computing Surveys 40(1): 1.1-1.39, http://dx.doi.10.1145/1322432. 1322433.

Apache Avro (2017) https://avro.apache.org (accessed 29/11/2017).

Apache Cassandra (2017) http://cassandra.apache.org (accessed 29/11/2017).

Apache Spark (2017) https://spark.apache.org/ (accessed 29/11/2017)

Batty M, Axhausen KW, Giannotti F et al. (2012) Smart cities of the future. European Physical Journal Special Topics 214(1): 481-518, http://dx.doi.10.1140/epjst/e2012-01703-3.

Bernstein D (2014) Containers and cloud: from LXC to Docker to Kubernetes. IEEE Cloud Computing 1(3): 81-84, http://dx.doi.org/10. 1109/MCC.2014.51.

Caragliu A, Del Bo C and Nijkamp P (2011) Smart cities in Europe. Journal of Urban Technology 18(2): 65-82, http://dx.doi.10.1080/ 10630732.2011.601117.

Casey G, Soga K, Silva E, Guthrie P and Kumar K (2017) A scalable agent based multi-modal modeling framework using real-time big-data sources for cities. In Proceedings of the Transportation Research Board 96th Annual Meeting. Transportation Research Board, Washington, DC, USA. No. 17-05941.

Cattell R (2010) Scalable SQL and NoSQL data stores. ACM Sigmod Record 39(4): 12-27, http://dx.doi.org/10.1145/1978915.1978919.
Chebotko A, Kashlev A and Lu S (2015), June. A big data modeling methodology for Apache Cassandra. In Proceedings of the 2015 IEEE International Congress on Big Data (BigData Congress) (Barbara C and Khan L (eds)). Institute of Electrical and Electronics Engineers, Washington, DC, USA, pp. 238-245.

Chen M, Mao S and Liu Y (2014) Big data: a survey. Mobile Networks and Applications 19(2): 171-209, http://dx.doi.10.1007/s11036-0130489-0.

Chourabi H, Nam T, Walker S et al. (2012) Understanding smart cities: an integrative framework. In Proceedings of the 2012 45th Hawaii International Conference on System Science (Sprague RH, Jr (ed.)). Institute of Electrical and Electronics Engineers, Washington, DC, USA, pp. 2289-2297.

CityDB (2017) https://www.3dcitydb.org (accessed 29/11/2017).

Edwards HC, Trott CR and Sunderland D (2014) Kokkos: enabling many core performance portability through polymorphic memory access patterns. Journal of Parallel and Distributed Computing 74(12): 3202-3216, http://dx.doi.org/10.1016/j.jpdc.2014.07.003.

Eisenacher C, Nichols G, Selle A and Burley B (2013) Sorted deferred shading for production path tracing. Computer Graphics Forum 32(4): 125-132, http://dx.doi.org/10.1111/cgf.12158.

Elasticsearch (2017) https://www.elastic.co/ (accessed 29/11/2017). Erlang (2017) https://www.erlang.org/ (accessed 29/11/2017).

Fabian N, Moreland K, Thompson D et al. (2011) The ParaView Coprocessing Library: A scalable, general purpose in situ visualization library. In 2011 IEEE Symposium on Large Data Analysis and Visualization, Providence, $R l$ (Rogers D and Silva CT (eds)). Institute of Electrical and Electronics Engineers, Washington, DC, USA, pp. 89-96.

Folk M, Heber G, Koziol Q, Pourmal E and Robinson D (2011) An overview of the HDF5 technology suite and its applications. In Proceedings of the EDBT/ICDT 2011 Workshop on Array Databases (Baumann P, Howe B, Orsborn K and Stefanova S (eds)). Association for Computing Machinery, New York, NY, USA, pp. $36-47$.

GraphX (2017) https://spark.apache.org/graphx/ (accessed 29/11/2017).

Hashem IAT, Yaqoob I, Anuar NB et al. (2015) The rise of 'big data' on cloud computing: review and open research issues. Information Systems 47: 98-115, http://dx.doi.org/10.1016/j.is.2014.07.006.

HDF5 (2017) https://support.hdfgroup.org/HDF5/ (accessed 29/11/2017). Hori M (2011) Introduction to Computational Earthquake Engineering, 2nd edn. Imperial College Press London, UK.

Johansen H, Rodgers A, Petersson NA et al. (2017) Toward exascale earthquake ground motion simulations for near-fault engineering analysis. Computing in Science \& Engineering 19(5): 27-37, http://dx. doi.org/10.1109/MCSE.2017.3421558.

Kale LV and Krishnan S (1993) CHARM++: a portable concurrent object oriented system based on C++. ACM Sigplan Notices 28(10): 91-108, http://dx.doi.org/10.1145/167962.165874.

Kitchin R (2014) The real-time city? Big data and smart urbanism. GeoJournal 79(1): 1-14, http://dx.doi.10.1007/s10708-013-9516-8. LeafletJS (2017) http://leafletjs.com/ (accessed 29/11/2017).

Lu X and Guan H (2017) Earthquake Disaster Simulation of Civil Infrastructures from Tall Buildings to Urban Areas. Springer, Singapore, Singapore.

Malewicz G, Austern MH, Bik AJ et al. (2010) Pregel: a system for largescale graph processing. In Proceedings of the 2010 ACM SIGMOD International Conference on Management of Data (Fletcher G (ed.)). Association for Computing Machinery, New York, NY, USA, pp. $135-146$.

Mozilla (2017) The WebGL API: 2D and 3D Graphics for the Web. Mozilla, Mountain View, CA, USA. See https://developer.mozilla.org/ en-US/docs/Web/API/WebGL_API (accessed 29/11/2017).

Open Street Map (2017) https://www.openstreetmap.org (accessed 29/11/2017) 
Smart Infrastructure and Construction

Volume 170 Issue SC4
Briefing: High-performance computing

for city-scale modelling and simulations

Soga, Casey, Kumar and Zhao
Palmer N, Miron E, Kemp R, Kielmann T and Bal H (2011) Towards collaborative editing of structured data on mobile devices. In 2011 IEEE 12th International Conference on Mobile Data Management, Lulea (Zaslavsky A, Chrysanthis PK, Lee DL et al. (eds)). Institute of Electrical and Electronics Engineers, Washington, DC, USA, pp. 194-199.

ParaView Catalyst (2017) https://www.paraview.org/in-situ/ (accessed 29/11/2017).

Pijanowski BC, Tayyebi A, Doucette J et al. (2014) A big data urban growth simulation at a national scale: configuring the GIS and neural network based land transformation model to run in a high performance computing (HPC) environment. Environmental Modelling and Software 51(2014): 250-268, http://dx.doi.org/10.1016/j.envsoft.2013. 09.015 .

Sánchez-Medina JJ, Galán-Moreno MJ and Rubio-Royo E (2010) Traffic signal optimization in 'La Almozara' district in Saragossa under congestion conditions, using genetic algorithms, traffic microsimulation, and cluster computing. IEEE Transactions on Intelligent Transportation Systems 11(1): 132-141, http://dx.doi.10. 1109/TITS.2009.2034383.

Singularity (2017) http://singularity.lbl.gov/ (accessed 29/11/2017).

Su K, Li J and Fu H (2011) Smart city and the applications. In Proceedings of the 2011 International Conference on Electronics, Communications and Control (ICECC). Institute of Electrical and Electronics Engineers, Washington, DC, USA, pp. 1028-1031.

Tadokoro S, Kitano H, Takahashi T et al. (2000) The robocup-rescue project: a robotic approach to the disaster mitigation problem.
In Proceedings of the 2000 IEEE International Conference on Robotics and Automation, ICRA 2000. Institute of Electrical and Electronics Engineers, Washington, DC, USA, vol. 4, pp. 4089-4094. Top500 (2017) https://www.top500.org/list/2016/11/ (accessed 29/11/2017).

Vicknair C, Macias M, Zhao Z et al. (2010) A comparison of a graph database and a relational database: a data provenance perspective. In Proceedings of the 48th Annual Southeast Regional Conference. Association for Computing Machinery, New York, NY, USA, p 42.

Waldrop MM (2016) The chips are down for Moore's law. Nature 530(7589): 144-147, http://dx.doi.org/10.1038/530144a.

Wijerathne MLL, Melgar LA, Hori M, Ichimura T and Tanaka S (2013) HPC enhanced large urban area evacuation simulations with vision based autonomously navigating multi agents. Procedia Computer Science 18(2013): 1515-1524, http://dx.doi.org/10.1016/j.procs.2013. 05.319 .

Yoshimura S, Hori M and Ohsaki M (2016) High-performance Computing for Structural Mechanics and Earthquake/Tsunami Engineering. Springer, Cham, Switzerland.

Zia K, Riener A, Farrahi K and Ferscha A (2012) A new opportunity to urban evacuation analysis: very large scale simulations of social agent systems in Repast HPC. In Proceedings of the 2012 ACM/IEEE/SCS 26th Workshop on Principles of Advanced and Distributed Simulation (Peng S (ed.)). Institute of Electrical and Electronics Engineers, Washington, DC, USA, pp. 233-242.

\section{How can you contribute?}

To discuss this paper, please email up to 500 words to the editor at journals@ice.org.uk. Your contribution will be forwarded to the author(s) for a reply and, if considered appropriate by the editorial board, it will be published as discussion in a future issue of the journal.

Proceedings journals rely entirely on contributions from the civil engineering profession (and allied disciplines). Information about how to submit your paper online is available at www.icevirtuallibrary.com/page/authors, where you will also find detailed author guidelines. 